\title{
Editorial comments for neoadjuvant chemo-radiotherapy in the treatment of locally advanced squamous cell esophageal cancer
}

\author{
Vadim G. Pischik ${ }^{1,2}$ \\ ${ }^{1}$ Department of Thoracic Surgery, Federal Hospital \#122, Saint Petersburg, Russia; ${ }^{2}$ Faculty of Medicine, Saint Petersburg State University, Saint \\ Petersburg, Russia \\ Correspondence to: Prof. Vadim G. Pischik, MD, PhD. 4 Prospect Kultury, Saint-Petersburg 194291, Russia. Email: vadim.pischik@mail.ru. \\ Comment on: Yang H, Liu H, Chen Y, et al. Neoadjuvant Chemoradiotherapy Followed by Surgery Versus Surgery Alone for Locally Advanced \\ Squamous Cell Carcinoma of the Esophagus (NEOCRTEC5010): A Phase III Multicenter, Randomized, Open-Label Clinical Trial. J Clin Oncol \\ 2018;36:2796-803.
}

Submitted Oct 15, 2018. Accepted for publication Oct 19, 2018.

doi: $10.21037 /$ jtd.2018.10.81

View this article at: http://dx.doi.org/10.21037/jtd.2018.10.81

In August 2018 issue of the Fournal of Clinical Oncology, Yang and coworker published a well-designed analysis of 451 patients with potentially resectable thoracic esophageal squamous cell carcinoma (SCC), clinically staged as T1-4N1M0/ T4N0M0, who were randomly assigned to the neoadjuvant chemoradiotherapy (NCRT) plus surgery, or to surgery alone groups (1). The study was presented as the phase III multicenter, randomized, open-label clinical trial. It was a great honor to be invited to provide editorial comments on this article.

Esophageal cancer (EC) is the fifth "oncological killer" worldwide as most common cause of cancer-related death in men and women (2). For the locally advanced EC, that is defined as T3-T4 or N1-3 M0, esophagectomy alone is not a standard treatment since a complete (R0) resection cannot be achieved in many cases. For that reason, neoadjuvant therapy is obviously indicated in operable patients with locally advanced disease (3).

It is well known, that prognosis and treatment results in patients with squamous cell (SCC) esophageal cancer are substantially worse than those for adenocarcinoma (AdC) (4). That is why some previous meta-analyses, where the patients with esophageal AdC and SCC were combined, showed inconsistency in terms of post-treatment outcomes (5). It should be noted, that commented investigation has very clear and strict inclusion criteria for the histology (just SCC) as well as for the EC stage (locally advanced disease). The authors did not specify what exactly staging system they have used, but they referred to the 6-th edition of AJCC Cancer Staging Manual. Because the time interval for this trial lasted from June 2007 to December 2014, we could speculate that staging systems they have used was a subject of changes. That could compromise the results to some extent, because the modern staging is based on the 8th edition of AJCC/UICC (6). Another question is about the patients with N2 and N3 disease, that could be included into the "locally advanced" group. Despite the authors claim that they ruled out this type of patients (they included just $\mathrm{N} 0-1$ patients), does it mean that the conclusion of this trial could be extrapolated onto whole locally advanced esophageal cancer?

The authors announced that NEOCRTEC5010 was conducted to clarify possible differences in the results for the Eastern Asia population. After reviewing this article we could conclude, that the results are in concordance with the last Americans and European randomized controlled trials (RCTs) for preoperative chemo-radiotherapy in EC patients (7). However, the investigated population was really different. Esophageal carcinoma is rare in young people and increases in incidence with age, getting the peak in the seventh and eighth decades of life (8). But in the commented study, some patients were 18 years old and $70 \%$ of the patients were younger than 60 years. This is unusual for European population. These differences potentially explain why the patients in the NEOCRTEC5010 trial, who are generally younger, might have better tolerated a preoperative chemo-radiotherapy.

Several RCTs have demonstrated a benefit in reducing local tumor recurrence and improving long-term survival 
(7,9-11). But not all of them have shown encouraging results. Duan et al., [2014] stated that among previous RCTs, that investigated the efficiency of NCRT compared to surgery, 6 trials did not show survival advantages (12). At the same time, despite the growing evidence that NCRT provides a survival benefit for EC patients, there is still a controversy over its impact on postoperative morbidity and mortality especially in elderly patients (13-15). Nevertheless, the sample sizes of previously conducted RCTs were relatively small, and it might have compromised their results. In NEOCRTEC501, 451 patients were recruited, that is, to the best of my knowledge, the biggest sample size for the trials on this topic. Second big advantage of the reviewed investigation is inclusion of mainly highvolume centers into the trial. Some meta-analyses suggest a benefit of centralization of esophageal cancer surgery to high-volume institutions in terms of mortality (16). Gabriel and co-authors [2018] have demonstrated that overall survival (OS) was superior for esophageal cancer patients treated at high throughput centers (17). Therefore, selection of such hospitals for NEOCRTEC5010 allowed to reduce the influence of the surgical experience on the results, providing the best possible ones.

An exceptionally impressive peculiarity of the study was a very low postoperative mortality and morbidity. Authors had reported just 2 postoperative deaths (1 patient in each group) out of the 412 totally operated patients. Sathornviriyapong and coauthors [2016], have analyzed eight RCTs that included 1058 locally advanced resectable esophageal SCC patients, and used meta-analysis to show that the NCRT with surgery group had a significantly higher rate of postoperative mortality and cardiopulmonary complications, but did not increase the risk of anastomosis and other complications, compared to the surgery-alone group (18). In NEOCRTEC5010, the only difference in postoperative morbidity between the compared groups was a statistically significant prevalence of arrhythmia in CRT patients. Surprisingly, anastomotic leakage and wound problems were more often seen in the surgery group. The differences were not statistically significant, but they would need to be explained. Probably there were some surgical details that could explain this but not explained in the text. It is impossible to understand from the paper what approaches were used for the esophagectomy. Was it minimally invasive or hybrid or standard open approach? On the other hand, the discussed trial has a very clear design. The groups were totally comparable and the inclusive criteria were comprehensive. Statistics and evaluations of the outcomes were transparent and understandable.

There are other interesting results, that have been demonstrated during subgroup analysis in this article. The best survival advantages with neoadjuvant CRT were observed in female patients and in those with T3 disease. Additional descriptors with near statistical significance in favor to neoadjuvant CRT were in N1 and middle third of esophageal tumor location.

It was suggested in the past, that preoperative CRT for esophageal SCC significantly increased patients' risk of cardiopulmonary complications and dropout before surgery (18). But this has not been the case when treatment is carried out in expert centers, with modern radiation planning techniques, use of adequate radiation doses and fractionation, and a good multidisciplinary cooperation and infrastructure (3). On the basis of the results of the Chemoradiotherapy for Oesophageal Cancer Followed by Surgery Study (CROSS) the weekly administration of carboplatin (doses titrated to achieve an area under the curve of $2 \mathrm{mg} / \mathrm{mL} / \mathrm{min}$ ) and paclitaxel $\left(50 \mathrm{mg} / \mathrm{m}^{2}\right.$ of bodysurface area) for 5 weeks and concurrent radiotherapy (41.4 Gy in 23 fractions, 5 days per week), followed by the surgery, could be recommended as a contemporary standard of care $(7,10)$. In the NEOCRTEC5010, vinorelbine $25 \mathrm{mg} / \mathrm{m}^{2}$ and cisplatin $75 \mathrm{mg} / \mathrm{m}^{2}$ were used with concurrent radiotherapy in a total dose of $40.0 \mathrm{~Gy}$ in 20 fractions of $2.0 \mathrm{~Gy}$, five fractions per week. The toxicity was precisely evaluated and stated, as well as the post-chemoradiation response and the tumor downstaging. There were additional tables showing compliance to chemotherapy with respect to dose density, adverse events and postoperative complications of patients receiving different cisplatin protocols, etc. The authors thoroughly described the patients from CRT group, who were not operated due to different reasons, but included all of them for overall survival analysis.

In conclusion, published phase III multicenter, randomized, open-label clinical trial gives a compelling evidence that neoadjuvant chemo-radiotherapy with planned surgery is considered to be the recommended definitive treatment for locally advanced SCC of the esophagus.

\section{Acknowledgements}

None.

\section{Footnote}

Conflicts of Interest: The author has no conflicts of interest to 
declare.

\section{References}

1. Yang H, Liu H, Chen Y, et al. Neoadjuvant Chemoradiotherapy Followed by Surgery Versus Surgery Alone for Locally Advanced Squamous Cell Carcinoma of the Esophagus (NEOCRTEC5010): A Phase III Multicenter, Randomized, Open-Label Clinical Trial. J Clin Oncol 2018;36:2796-803.

2. Torre LA, Bray F, Siegel RL, et al. Global cancer statistics, 2012. CA Cancer J Clin 2015;65:87-108.

3. Lordick F, Mariette C, Haustermans K, et al. Oesophageal cancer: ESMO Clinical Practice Guidelines for diagnosis, treatment and follow-up. Ann Oncol 2016;27:v50-7.

4. Pennathur A, Gibson MK, Jobe BA, Luketich JD. Oesophageal carcinoma. Lancet 2013;381:400-12.

5. Deng J, Wang C, Xiang M, et al. Meta-analysis of postoperative efficacy in patients receiving chemoradiotherapy followed by surgery for resectable esophageal carcinoma. Diagn Pathol 2014;9:151.

6. Rice TW, Patil DT, Blackstone EH. 8th edition AJCC/UICC staging of cancers of the esophagus and esophagogastric junction: application to clinical practice. Ann Cardiothorac Surg 2017;6:119-30.

7. Shapiro J, van Lanschot JJ, Hulshof MC, et al. Neoadjuvant chemoradiotherapy plus surgery versus surgery alone for oesophageal or junctional cancer (CROSS): long-term results of a randomised controlled trial. Lancet Oncol 2015;16:1090-8.

8. Rustgi AK, El-Serag HB. Esophageal carcinoma. N Engl J Med 2014;371:2499-509.

9. Speicher PJ, Wang X, Englum BR, et al. Induction chemoradiation therapy prior to esophagectomy is associated with superior long-term survival for esophageal cancer. Dis Esophagus 2015;28:788-96.

Cite this article as: Pischik VG. Editorial comments for neoadjuvant chemo-radiotherapy in the treatment of locally advanced squamous cell esophageal cancer. J Thorac Dis 2018;10(11):5979-5981. doi: 10.21037/jtd.2018.10.81
10. van Hagen P, Hulshof MC, van Lanschot JJ, et al. Preoperative chemoradiotherapy for esophageal or junctional cancer. N Engl J Med 2012;366:2074-84.

11. Tepper J, Krasna MJ, Niedzwiecki D, et al. Phase III trial of trimodality therapy with cisplatin, fluorouracil, radiotherapy, and surgery compared with surgery alone for esophageal cancer: CALGB 9781. J Clin Oncol 2008;26:1086-92.

12. Duan XF, Tang P, Yu ZT. Neoadjuvant chemoradiotherapy for resectable esophageal cancer: an in-depth study of randomized controlled trials and literature review. Cancer Biol Med 2014;11:191-201.

13. Hamai Y, Hihara J, Taomoto J, et al. Effects of neoadjuvant chemoradiotherapy on postoperative morbidity and mortality associated with esophageal cancer. Dis Esophagus 2015;28:358-64.

14. Mungo B, Molena D, Stem M, et al. Does neoadjuvant therapy for esophageal cancer increase postoperative morbidity or mortality? Dis Esophagus 2015;28:644-51.

15. Ruol A, Portale G, Castoro C, et al. Effects of neoadjuvant therapy on perioperative morbidity in elderly patients undergoing esophagectomy for esophageal cancer. Ann Surg Oncol 2007;14:3243-50.

16. Markar SR, Karthikesalingam A, Thrumurthy S, Low DE. Volume-outcome relationship in surgery for esophageal malignancy: systematic review and meta-analysis 20002011. J Gastrointest Surg 2012;16:1055-63.

17. Gabriel E, Narayanan S, Attwood K, et al. Disparities in major surgery for esophagogastric cancer among hospitals by case volume. J Gastrointest Oncol 2018;9:503-16.

18. Sathornviriyapong S, Matsuda A, Miyashita M, et al. Impact of Neoadjuvant Chemoradiation on ShortTerm Outcomes for Esophageal Squamous Cell Carcinoma Patients: A Meta-analysis. Ann Surg Oncol 2016;23:3632-40. 